\title{
Craniomaxillofacial Allotransplantation: Achievements and Future Challenges
}

\author{
Devin Coon $\cdot$ Srinivas M. Susarla $\cdot$ Chad R. Gordon
}

Published online: 18 December 2012

(C) Springer Science + Business Media New York 2012

\begin{abstract}
Traditional reconstructive techniques have struggled to restore form and function in cases of massive facial trauma. A growing number of centers are attempting to make reconstructive craniomaxillofacial transplantation a mainstream technique. In this chapter, we review the worldwide clinical experience and lessons learned from previous transplants. Increasing efforts are being applied towards the use of computer-based surgical and orthognathic planning to mitigate the inherent complexity of these cases. As comfort is gained with the technical aspects of harvest, inset, and anastomosis, the benchmark for success will be moved beyond allograft survival into measurements of functionality and appearance.
\end{abstract}

Keywords LeFort osteotomy - Craniomaxillofacial allotransplantation · Face transplant · Maxillofacial allotransplantation - Composite tissue allograft . Orthognathic surgery · Craniofacial surgery ·

Vascularized composite allotransplantation

\section{Introduction}

Traditional autologous tissue-based reconstructive techniques have struggled to reliably restore form and function in

D. Coon · C. R. Gordon $(\bowtie)$

Department of Plastic and Reconstructive Surgery, The Johns Hopkins Medical Institutions, Johns Hopkins University School of Medicine, 801 N. Caroline Street, 8th Floor, Baltimore MD 21287, USA

e-mail: cgordon@jhmi.edu

S. M. Susarla

Department of Oral and Maxillofacial Surgery, Massachusetts General Hospital, Harvard School of Dental Medicine, Boston, MA, USA cases of massive soft tissue and bony facial trauma. With the achievement of the first successful face and maxilla allotransplant in 2008, increasing interest has been focused on the potential of transplantation to provide superior aesthetic and functional outcomes for these patients [1,2]. A growing number of centers worldwide are attempting to convert maxillofacial transplantation from an experimental procedure to a mainstream reconstructive technique [3]. Over the coming years, vascularized composite allotransplantation (VCA) is likely to become an increasingly important tool to the reconstructive surgeon, and familiarity with its basic tenets is valuable [4]. In this chapter, we focus on principles and developments in osteocutaneous craniomaxillofacial (CMF) transplantation.

\section{Clinical Experience}

The initial report of osteocutaneous facial transplantation was in 2006 by Zhang et al. [5] in China. A 30-year-old man who had been mauled by a bear underwent CMF transplantation from a 25 -year-old male donor. The allotransplant included the skin of the right cheek and upper lip and nose as well as the right parotid gland and bony segments of the right anterior maxillary sinus and lateral orbital rim. The initial operation was a success, though the authors noted several aspects for future technical refinement (e.g., enlarged orbital volume leading to the need for orbital floor reconstruction). However, the patient developed immunosuppressive medication-related hyperglycemia on day 3 and remained on insulin therapy for 21 months before dying at 24 months post-transplant. Though the etiology of death is unclear, it has generally been attributed to non-compliance with immunosuppression.

The next attempt at CMF transplantation was by the Cleveland Clinic team in 2008 [1]. This was a LeFort III- 
based osteomyocutaneous face and maxilla transplant, reconstructing a close-range, ballistic midfacial defect in a 45 -year-old woman. The patient had previously undergone numerous attempts at autologous reconstruction: (1) temporalis muscle transposition, (2) radial forearm free flap, (3) anterolateral thigh flap, (4) osteocutaneous free fibula flap, (5) split thickness calvarial bone grafts, (6) forehead flap, and (7) multiple skin grafts. The allograft was a composite LeFort-III based midfacial skeletal allotransplant including overlying skin, soft tissue, the entire nose, lower eyelids, upper lip, bilateral zygomas/orbital floor, bilateral parotid glands, anterior maxilla with alveolar process and maxillary incisors, anterior hard palate, and oral mucosa. The neurovascular pedicles included the bilateral external carotid arteries, external jugular/posterior facial veins, and bilateral facial nerves with a total operative time of $22 \mathrm{~h}$. To date, there have been no major complications reported in this recipient. Revisional surgeries included bilateral ectropion repair and neck skin excision. A palatal obturator has been used to fill a palatal defect at the interface of the donor and recipient palates.

Neurosensory testing at 3 months post-operatively demonstrated re-innervation of the transplanted skin with normal two-point discrimination at 1 year. Facial mimetics were noted to be improved at 6 months, with a symmetric smile and steady improvement in upper lip and lower eyelid movements. The patient has had significant improvement in speech, nasal breathing, olfaction, and taste. Mastication and swallowing improved and soft foods/ liquids were tolerated at 6 months post-surgery. However, there was a significant class II skeletal profile, with mandibular retrognathism and excessive overjet (Fig. 1). This most likely occurred due to the longer maxilla of the donor and size mismatch in comparison to the recipient's facial skeleton, highlighting the potential for application of traditional orthognathic concepts to improve outcomes.

Three additional osteocutaneous CMF transplants have been reported. Lantieri performed transplantation of the premaxilla, chin, nose, skin, and bilateral parotid glands in a 28-year-old gunshot victim [6]. Bilateral facial and trigeminal nerves were used with vascular anastomosis to bilateral external carotid arteries and facial veins with a total operative time of $15 \mathrm{~h}$. The patient has reportedly experienced an uncomplicated postoperative course. Pomahac et al. reported the transplantation of a dentate maxilla/hard palate with associated skin and facial mimetic muscles, nose, and upper lip in a 59-year-old man after an electrical burn injury. Arterial anastamoses included the facial artery (right) and external carotid artery (left). The donor's external jugular vein was anastamosed to the recipient's facial vein with a total operative time was $17 \mathrm{~h}$.

Most recently, Rodriguez et al. [7] performed the first double-jaw CMF transplant in 2012. A 37-year-old man

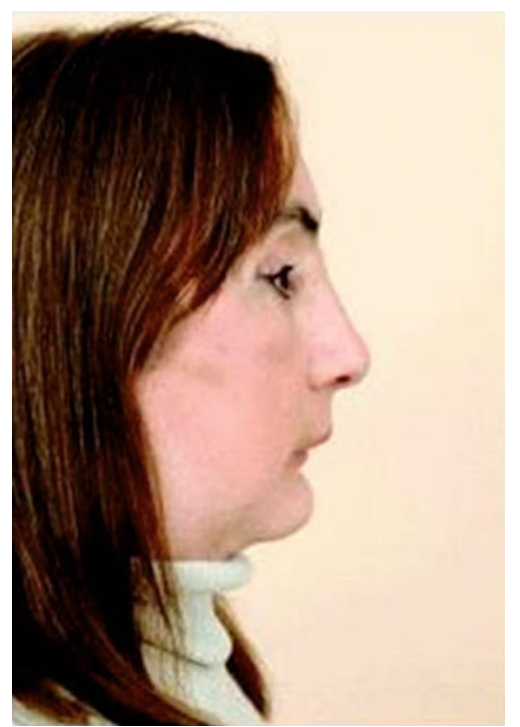

Fig. 1 The Cleveland Clinic maxillofacial transplant recipient displays post-operative mandibular retrognathia and class II occlusion. (From Siemionow et al. [16]; copyright 2009. Reproduced with permission from The Lancet Publishing Group.)

with ballistic gunshot injuries underwent transplantation including the dentate maxilla and mandible as well as a portion of the tongue. Again, the patient's postoperative course was uncomplicated, suggesting that the growing worldwide experience with CMF transplantation is helping to improve outcomes.

\section{Integration of Orthognathic Planning}

With increasing success maintaining graft survival, more attention is being paid to functional and aesthetic outcomes such as skeletal relation, hybrid occlusion, and facial harmony. There are typically approximately $24 \mathrm{~h}$ between donor identification and transplantation in which surgical planning must occur. We propose that orthognathic planning should be considered an essential part of this process. The first step is analysis of the maxillofacial skeletal profile of the recipient and donor to identify pre-operative facial form and function. Detailed evaluation of the maxillomandibular relationships should be undertaken, with measurements focusing on the multi-dimensional relationships between the skeletal and dental landmarks. This analysis is essential not only for determination of dental arch compatibility but also for including orthognathic corrections into the transplant procedure. Figure 2 illustrates what we consider to be some of the essential components to consider in the initial orthognathic evaluation [8].

Dental cast models are generated for both the recipient and donor jaws. Using these models, the donor maxilla can be manually articulated with the recipient mandible and any size mismatch can be evaluated. Models are placed on 
a Giletti articulator to evaluate best-fitting occlusion with occlusal grinding as needed for maximum intercuspation. An acrylic splint is fashioned to assist with intraoperative inset of the alloflap (Fig. 3). If the recipient or donor are edentulous, gunning-type splints can be used to provide the necessary height spacing for future dental restoration, with maxillomandibular fixation screws for fixation guidance.

The relationship between the maxillary and mandibular arches is evaluated using standard cephalometric landmarks (e.g., SNA, SNB) as well as dental relation as per our previous reports [8,9••]. An understanding of stability in orthognathic surgery is essential in understanding the limitations of post-transplant corrections. Figure 4 represents our current algorithm for pre-operative planning in osteocutaneous transplantation of the midface.

It should be emphasized that the vertical facial dimension is of equal importance and must not be neglected. Cephalometric analysis is valuable in the initial assessment of discrepancies between the donor and recipient. The orbital and nasofrontal positioning that would result from use of the articulated models should be evaluated. If the positioning of the naso-orbital segments leads to vertical maxillary excess, rotation of the mandible should be taken into account and simulated to identify whether there is potential for an open-bite deformity to occur. Optimal hybrid occlusion includes strong posterior contact bilaterally and midline centric occlusion/relation.

\section{Cadaveric Studies}

During the early clinical development of CMF VCA, cadaveric models have provided invaluable insight into a variety of different aspects, ranging from technical feasibility to comparisons with free tissue transfer. One area of interest to both patients and surgeons has been the concept of "identity transfer", i.e. the degree to which the transplant recipient's face comes to resemble that of the donor. Unlike myocutaneous alloflaps, where the recipient's facial skeleton largely determines the final appearance, Siemionow et al. [10] found that LeFort-based osteocutaneous alloflaps produce a hybrid appearance with a significant portion of the
Fig. 2 Orthognathic factors to consider in facial transplantation planning. (From Gordon et al. [8]; copyright 2012. Reproduced with permission from Lippincott Williams and Wilkins.)

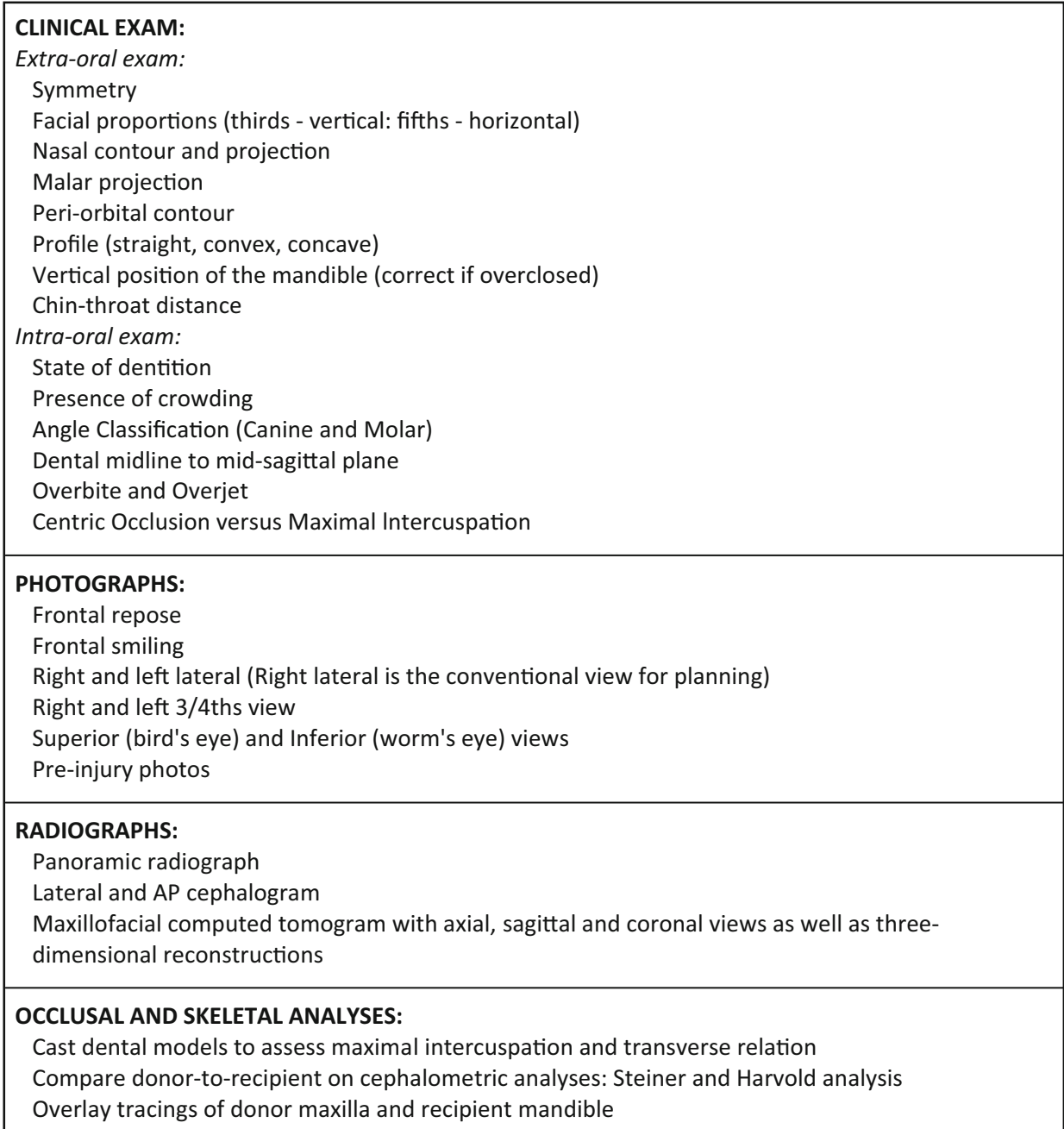




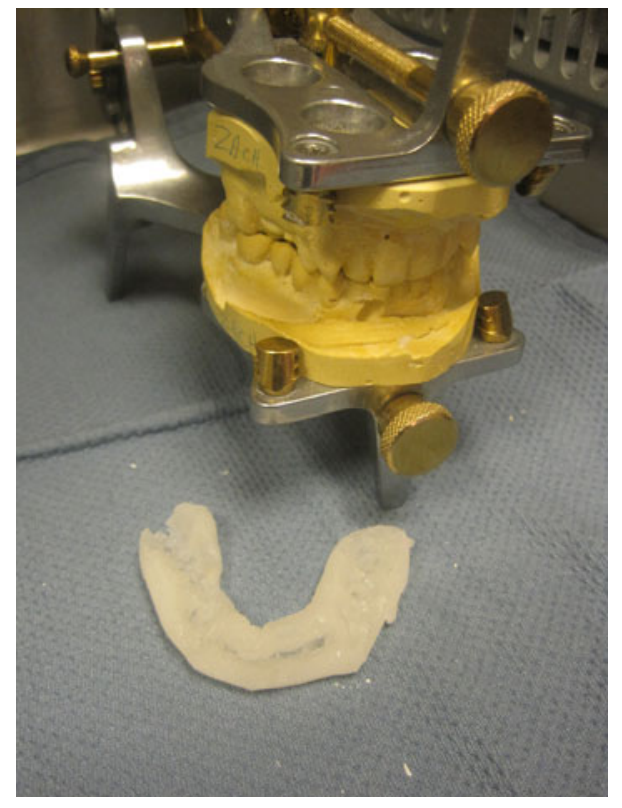

Fig. 3 The hybrid splint is created after selective occlusal grinding of cast models during a cadaveric mock face transplantation. (From Gordon et al. [9••]; copyright 2011. Reproduced with permission from Lippincott Williams and Wilkins.)

donor's features remaining. These studies on osteocutaneous CMF transplantation led to recognition that cadaveric models could be valuable in assessing the orthognathic variables that affect both appearance and function postoperatively.

Baccarani et al. [11] assessed cadaveric alloflap harvest techniques. Myocutaneous flap harvest was performed with a subgaleal, sub-SMAS, and sub-platysmal dissection while osteomyocutaneous flap harvest was achieved via a subperiosteal approach. This provided support for the feasibility of simultaneous bone and soft tissue reconstruction via inclusion of the vascularized midface. This technical approach was eventually utilized by the Cleveland Clinic team in their facial transplant.

Yazici et al. analyzed LeFort II-based osteocutaneous face transplantation. They examined the vascular territories of the face with an emphasis on defining ideal alloflap harvest designs [12]. The masticatory and pterygomaxillary spaces were found to contain the key vascular structures for their approach. Pomahac also performed similar vascular studies in a LeFort III model, finding that the facial artery system appeared to have retrograde supply of the maxilla adequate to support transplantation [13•]. In contrast, however, the deep compartments of the cheek and masticatory muscles were supplied by maxillary artery branches, and therefore they felt that these could not be safely included in a flap based on a facial arterial blood supply.

Banks et al. [14] conducted similar LeFort III-based cadaveric injection studies. They found that the skin of the entire midface could be supplied based on a single external carotid vessel. However, they observed that only one side of the face was perfused at the level of bone and mucosa. Furthermore, while the lower maxilla and alveolus were well supplied by the facial artery, there was relatively little perfusion of the upper maxilla and zygoma. They therefore raised concerns about whether preservation of the internal maxillary branch of the external carotid would be necessary to support these bony segments. However, latex injection studies have a limited ability to define microscopic anastomotic networks; in the Cleveland Clinic transplant, this did not appear to be a clinically significant problem.

Our group then sought to expand the scope of cadaveric CMF allotransplantation models to include orthognathic considerations [9••]. Three mock face transplants were performed in fresh cadaveric heads using a LeFort III model incorporating all the skeletal and soft tissue components of the midface. The first (MFT-1) used the previously performed Cleveland Clinic technique from a dentate recipient to a dentate donor without additional orthognathic assessment. The second (MFT-2) used a similar scenario, but with orthognathic planning and application to assess changes in technique and outcomes. Alginate impressions were obtained to evaluate alterations, and the resulting models were placed on a Giletti articulator. Occlusal grinding was performed to establish an ideal occlusion, and a hybrid acrylic occlusal splint was fabricated using the donor maxilla and recipient mandibular occlusal planes. During the transplant procedure, the splint was used to position the jaws after which maxillo-mandibular fixation was applied. Rigid miniplate fixation of the LeFort III segments was then performed bilaterally at the naso-frontal, zygomatico-frontal, and zygomatico-temporal sites. The third transplant model (MFT-3) represented an edentulous transplant. IMF screws were used in combination with an impression tray to set vertical spacing.

While there are many similarities between traditional orthognathic principles and their application in CMF transplantation, there are also conceptual differences that are still being defined. The classic Angle definition of proper occlusion as the mesiobuccal cusp to mesial groove of the first molar cannot be applied, as the dimensions of the donor maxilla to recipient mandible are inherently dissimilar. Instead, for dentate transplantations, we have chosen to define ideal hybrid occlusion as the "presence of bilateral posterior contact with $<2 \mathrm{~mm}$ of overbite and overjet with midline symmetry".

We found that, as in the Cleveland Clinic living recipient, transplantation without orthognathic planning (MFT-1) produced malocclusion with 4-5 $\mathrm{mm}$ overjet and a class II occlusion. In the MFT-2 case, pre-operative assessment demonstrated the donor to have a class III skeletal relationship while the recipient was class II, emphasizing the inherent asymmetries that commonly 


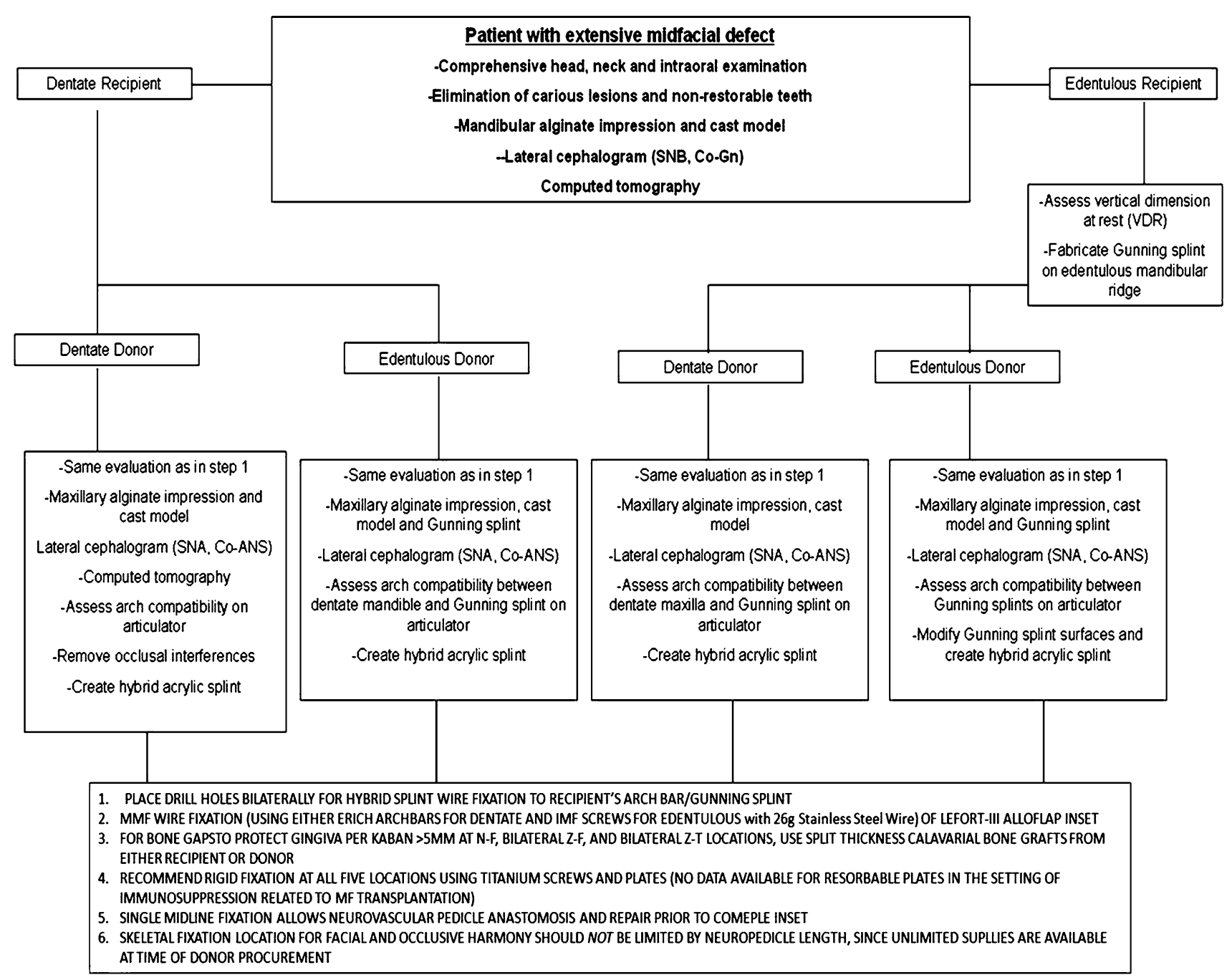

Fig. 4 Our current algorithm for occlusal and skeletal planning in the maxillofacial transplant patient. (From Gordon et al. [9••]; copyright 2011. Reproduced with permission from Lippincott Williams and Wilkins.)

result if orthognathic considerations are not addressed. However, utilization of the hybrid splint to position the maxilla resulted in gapping at the naso-frontal junction as well as inadequate orbital volume. We therefore repositioned the flap, accepting several millimeters of posterior vertical excess in exchange for maintenance of adequate orbital volume and overall facial harmony. This resulted in an anterior open bite of $1.7 \mathrm{~mm}$. Overall, the hybrid result demonstrated skeletal relationship comparable to class I, which represented an improvement for both the donor and recipient in terms of overall appearance (Fig. 5).

This experience was valuable in emphasizing the important point that, unlike in standard orthognathics, establishing correct occlusion does not necessarily provide the ideal framework for fixation: the donor maxilla and recipient mandible are not proportional and the final positioning represents a compromise between competing factors. This led us to attempt to elucidate specific principles to consider during flap inset and their relative priorities. In order of descending priority, these are:

1) Establishment of sagittal relationship of the midface skeleton - precise sagittal positioning of the midface segment is vital for aesthetic appearance and airway patency.

2) Preservation of ideal orbital volume with orbital floor reconstruction - enophthalmos is a highly challenging problem to correct with significant aesthetic and ophthalmological consequences. It is essential for the orbital volume to be correct at the time of transplantation.

3) Minimization of bony gaps between the donor and recipient bony segments-donor bone grafts can be utilized as needed for small gaps; however, to obtain the needed bony union, it is essential that large gaps be avoided.

4) Maintenance of vertical maxillary alignment relative to the upper facial skeleton-for aesthetic and functional 


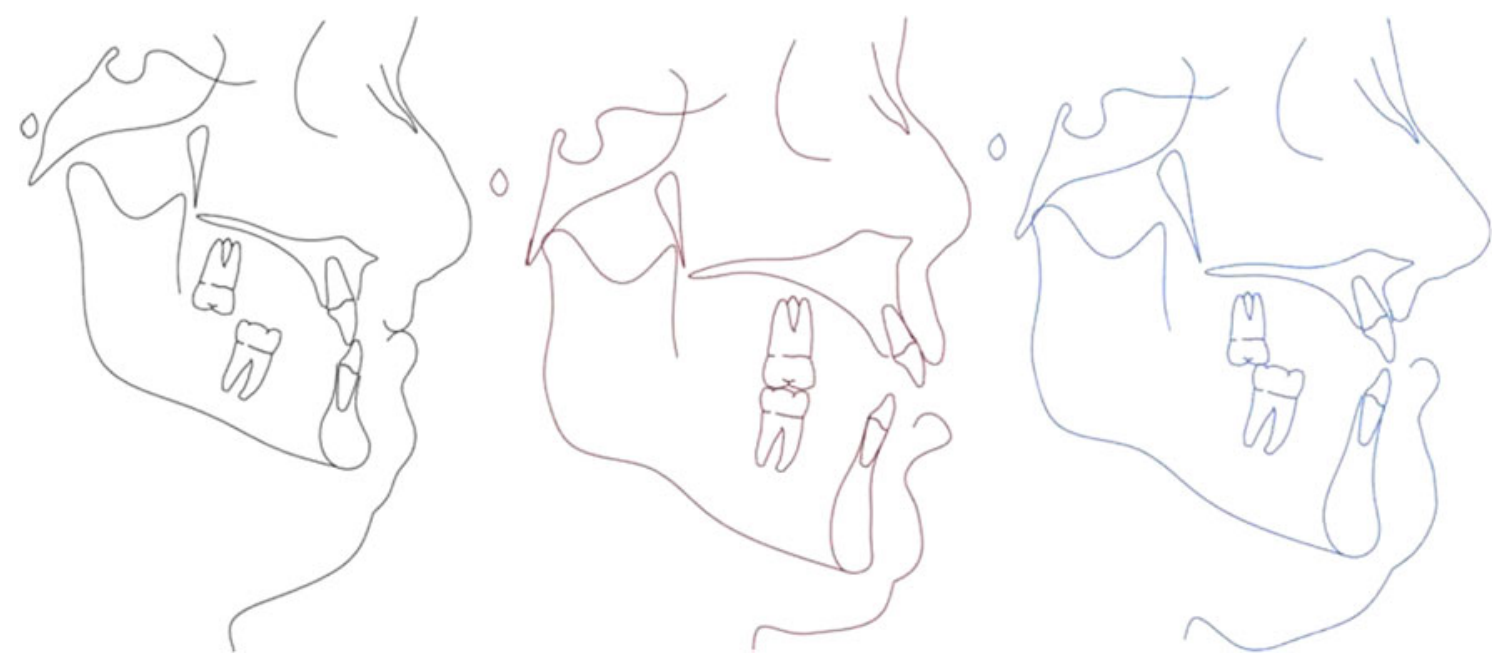

Fig. 5 Cephalometric analysis for the dentate cadaveric maxillofacial transplantation model demonstrates the pre-operative donor (left), pre-defect creation recipient (center), and post-transplant recipient

purposes, the appropriate maxillary height must be maintained.

5) Establishment of dental contact points-for the purposes of mastication, one anterior and two posterior contact points should be established, with bilateral posterior points being the higher priority of the two.

A different set of considerations is posed by a transplant where the alloflap contains both jaws. While more demanding from the perspective of harvesting and inset, the orthognathic considerations posed are somewhat simpler. In preparation for their double-jaw allotransplantation, Brown et al. conducted a study of 10 cadaveric transplants. Surgical modeling was used to plan the procedure, with pre-fabricated cutting guides for the recipient osteotomies [15•]. Cutting guides were not used for the donor in consideration of the fact that the window between donor identification and harvest is unlikely to provide sufficient time for fabrication. In contrast to previous alloflaps containing the mandible, bilateral sagittal split osteotomy (BSSO) was performed to allow more flexible positioning of the mandibular segment. Post-operative cephalometric analysis demonstrated no significant difference between the values predicted by the planning software and the actual transplant recipient values. This demonstrates the value of computer-based surgical planning in minimizing the need for "on-table" modifications and decreasing variability in outcomes.

\section{Conclusions and Future Work}

Several areas appear to represent likely avenues of future technological improvement. The incorporation of (right). (From Gordon et al. [9••]; copyright 2011. Reproduced with permission from Lippincott Williams and Wilkins.)

computer-aided preoperative planning has been shown to facilitate alloflap design and improve reliability. Computer algorithms to analyze and compensate for the mismatch between donor and recipient jaws have the potential to iteratively optimize the flap inset process in midface transplantation. Advances in on-demand 3-dimensional printing may allow for cutting guides to be produced rapidly enough for use in both donor and recipient osteotomies. Customized plate technology which is pre-contoured based on the planned osteotomies may also find a role.

Progress within the field of human facial allotransplantation shows no signs of slowing. As increasing comfort is gained with the technical aspects of harvest, inset, and anastomosis, the benchmark for success will be moved beyond allograft survival into measurements of functionality and appearance. Adaptation and inclusion of traditional orthognathic principles into the surgical planning process has the potential to improve the masticatory and aesthetic outcomes of CMF transplantation.

Disclosure No potential conflicts of interest relevant to this article were reported.

\section{References}

Papers of particular interest, published recently, have been highlighted as:

- Of importance

-. Of major importance

1. Alam DS, Papay F, Djohan R, et al. The technical and anatomical aspects of the World's first near-total human face and maxilla transplant. Arch Facial Plast Surg. 2009;11(6):369-77. 
2. Coffman KL, Gordon C, Siemionow M. Psychological outcomes with face transplantation: overview and case report. Curr Opin Organ Transplant. 2010;15(2):236-40.

3. Siemionow M, Gordon CR. Overview of guidelines for establishing a face transplant program: a work in progress. Am J Transplant. 2010;10(5):1290-6.

4. Siemionow MZ, Zor F, Gordon CR. Face, upper extremity, and concomitant transplantation: potential concerns and challenges ahead. Plast Reconstr Surg. 2010;126(1):308-15.

5. Guo S, Han Y, Zhang X, et al. Human facial allotransplantation: a 2-year follow-up study. Lancet. 2008;372(9639):631-8.

6. Lantieri L, Hivelin M, Audard V, et al. Feasibility, reproducibility, risks and benefits of face transplantation: a prospective study of outcomes. Am J Transplant. 2011;11(2):367-78.

7. Bojovic B, Dorafshar AH, Brown EN, et al. Total face, double jaw, and tongue transplant research procurement: an educational model. Plast Reconstr Surg. 2012;130(4):824-34.

8. Gordon CR, Susarla SM, Peacock ZS, Kaban LB, Yaremchuk MJ. LeFort-based maxillofacial transplantation: current state of the art and a refined technique using orthognathic applications. J Craniofac Surg. 2012;23(1):81-7.

9. • Gordon CR, Susarla SM, Peacock ZS, et al. Osteocutaneous maxillofacial allotransplantation: lessons learned from a novel cadaver study applying orthognathic principles and practice. Plast Reconstr Surg. 2011;128(5):465e-79e. This cadaveric study performed several simulated maxillofacial allotransplants. It was demonstrated that preoperative orthognathic planning led to significantly more harmonious results compared to intraoperative adjustment alone. New algorithms for integration of these concepts were proposed and tested.
10. Siemionow M, Agaoglu G. The issue of "facial appearance and identity transfer" after mock transplantation: a cadaver study in preparation for facial allograft transplantation in humans. J Reconstr Microsurg. 2006;22(5):329-34.

11. Baccarani A, Follmar KE, Baumeister SP, Marcus JR, Erdmann D, Levin LS. Technical and anatomical considerations of face harvest in face transplantation. Ann Plast Surg. 2006;57(5):483-8.

12. Yazici I, Cavusoglu T, Comert A, et al. Maxilla allograft for transplantation: an anatomical study. Ann Plast Surg. 2008;61(1):105-13.

13. - Pomahac B, Lengele B, Ridgway EB, et al. Vascular considerations in composite midfacial allotransplantation. Plast Reconstr Surg. 2010;125(2):517-22. Cadaveric dissections demonstrated an anastomotic network present between the facial and maxillary vessels around the midfacial skeleton. This anatomic feature was used by the authors as the basis for supplying one side of their maxillofacial transplant on the facial artery alone.

14. Banks ND, Hui-Chou HG, Tripathi S, et al. An anatomical study of external carotid artery vascular territories in face and midface flaps for transplantation. Plast Reconstr Surg. 2009;123(6):1677-87.

15. - Brown EN, Dorafshar AH, Bojovic B, et al. Total face, double jaw, and tongue transplant simulation: a cadaveric study employing computer-assisted techniques. Plast Reconstr Surg. 2012;130(4):815-23. Twenty double-jaw maxillofacial allotransplants were performed in cadavers. Use of surgical planning software with custom cutting guides allowed easier inset and postoperative results were nearly identical to planning software predictions.

16. Siemionow M, Papay F, Alam D, et al. Near-total human face transplantation for a severely disfigured patient in the USA. Lancet. 2009;374:203-9. 